\title{
Method for Shadow Removal of Moving Object in YUV Color Space
}

\author{
Yongsheng $\mathrm{Hu}^{\text {a }}$, Liyong Nan ${ }^{\mathrm{b}}$ \\ School of Information Engineering, Binzhou University, Binzhou, Shandong 256603, China \\ ahuys1208@163.com, bclodhopper2008@163.com
}

\begin{abstract}
Keywords: YUV; Color Space; Moving Object Detection; Shadow Detection; Shadow Removal.
\end{abstract}
\begin{abstract}
In the course of moving object detection, the shadow of objects may arise many problems, such as the distortion of the object shape, the adhesion between different objects and so on. To cope with these problems, a fast shadow removal method based on video image is proposed. By the computing model based on YUV color space, the distortion coefficient between moving object and background can be obtained, and then the shadow can be removed by this value. Finally, experimental results show that the method for shadow removal can detect the shadow and separate several joined objects correctly.
\end{abstract}

\section{Introduction}

With the development of computer vision technology, electronic technology and communications technology development, intelligent video surveillance system has been widely used in various occasions. The detection of moving object is the first process of intelligent video surveillance system. The quick and correct detection of moving object can provide favorable conditions to subsequent object segmentation, object tracking, classification and recognition et al. [1].However, light has great influence on video image, for example, different intensities and different angles of light irradiation exposed on translucent or opaque objects may cast shadows. Due to shadows with the same motion characteristics of object itself, so the shadows will be incorrectly detected as part of the moving object. Existence of shadows in moving object detection process can result in reducing accuracy of object detection, distorting the object shape, the adjacency between different objects etc, which can cause very serious problems to subsequent processing. Shadow detection and removal is the problem to be solved urgently in intelligent video surveillance system.

Shadow detection methods mainly include two types: model-based and property-based. Model-based methods[2][3] are based on matching sets of geometric features and rely on models representing the priori knowledge of the geometry of the scene, the objects, and the light source. Because of these limitations, model-based methods are only applicable to the special occasions. Property-based methods are using the specific shadow properties such as edges, brightness, texture, different color spaces to separate shadow from the object and can obtain better result. Angie WKS et al.[4] used the boundary information to remove shadows in traffic video. Because shadow didn't change the background texture, Hoang $\mathrm{M}$ A et al.[5] presented a texture-based method for object extraction. This method can remove shadow directly, but it is computation-intensive and its threshold value was difficult to obtain. Using optical properties of shadow, and combining its texture feature, Kollerd et al.[6] proposed a shadow detection method by region growing. And the detection result is accurate in the case of moving object had simple and normal texture feature. Liu Yang et al.[7]proposed a shadow suppression method based on chrominance distortion and texture, but the method didn't apply in condition of low brightness and high saturation. Cucchiara et al.[8] advocated to use HSV space to remove shadow, and the main reason was that the brightness and chromaticity had been separated in HSV color space. By analyzing the characteristics of shadow and background in HSV color space, Zhang Xia et al.[9] used the different characteristics of shadow and moving object in $\mathrm{H}, \mathrm{S}, \mathrm{V}$ components, then computed its corresponding threshold value and eliminated shadow by the value. Fu Ping et al.[10] developed a method based on illumination assessment. Multi-gradient analysis and a fast clustering algorithm for the shady image are employed to detect the shadow. The 
method can detect and remove very light shadows. Liu Xue et al.[11]put forward a shadow suppression method based on chrominance distortion in YUV color space and first order gradient information. Ma Guofeng et al.[12]combined chroma and intensity to remove shadows in YUV color space.

All the shadow removal methods mentioned above can be successful under respective given conditions. Their basic idea is to detect motion areas firstly, then separate shadows from motion areas and last remove shadows to get final moving objects. This paper proposes a shadow removal method based on video image. The method can detect moving objects and remove shadows simultaneously in video images. The method calculates the distortion coefficient between moving object and background by the computing model based on YUV color space, and then the shadow can be removed by this value.

The paper is organized as follows. Part 2 firstly analyzes forming mechanism of shadow, and then presents pixel calculation model, finally proposes shadow removal method. The experimental results and comparisons are analyzed and shown in part 3. Finally, Part 4 concludes the paper.

\section{Shadow Detection and Removal}

\subsection{Shadow Analysis}

Shadow has two important visual features: 1) There are obvious differences between shadow and background; 2) Moving object and its shadow has the same motion characteristics. So there is much chance that object shadow is often erroneously detected as foreground. It is urgent to separate shadow from moving object in practical application system. Shadow removal methods based on shadow properties detect and remove shadow according to changes of pixel information in shadow areas.

Output format of video image is RGB or YUV. RGB format is a mixture of three primary colors (Red, Green and Blue) in a different proportion; three components of YUV format describes brightness, chromaticity and intensity information of an image. YUV color space has already separated brightness and chromaticity, so it occupied little bandwidth compared with RGB color space. Taking into account computational efficiency and purposes of shadow detection, YUV color space was selected in our research. Removing shadow in YUV color space is generally based on the following hypothesis ${ }^{[13]}: 1$ ) Brightness of cast shadows is lower than the background; 2) The chromaticity of cast shadows is equal or nearly the same as background.

A fast shadow removal method based on video image is proposed on the basis of visual features of shadow and hypothesis of removing shadow in YUV color space. The basic idea of the method is: 1) Extract the brightness component of pixel point and give it an appropriate weight depending on the brightness differences between objective shadow pixel and background pixel; 2) Extract the chromaticity and intensity component of the pixel point, and then detect shadow according to the same or little difference chromaticity and intensity between objective shadow pixel and background pixel.

\subsection{Pixel Computing Model and Shadow Removal Method}

Let YUV value of objective pixel point $F$ in an image be the vector EF(EFx, EFy, EFz), YUV value of shadow pixel point I be the vector(EIx, EIy, EIz), and YUV value of background pixel point B be the vector $\mathrm{EB}(\mathrm{EBx}, \mathrm{EBy}, \mathrm{EBz})$, which are shown in Fig.1. These three vectors respectively describe the information of object, objective shadow, background on the aspect of brightness, chromaticity and intensity.

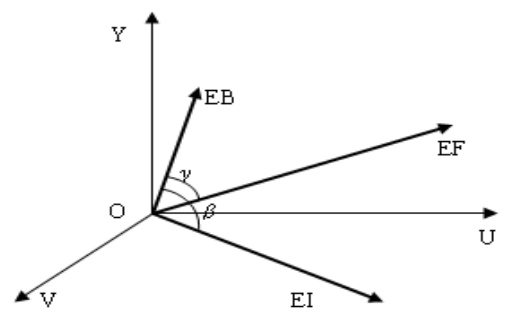

Fig.1 the pixel point representation of object, objective shadow, and background in YUV space 
As shown in Fig. 1, $\gamma$ is the angle between vector EB and vector EF, $\beta$ is the angle between vector EB and vector EI. $\gamma$ is called the distortion coefficient of object background, $\beta$ is called the distortion coefficient of objective shadow background. Vector EF and EI are defined as moving object vector EM (EMx, EMy, Emz), distortion coefficient $\gamma$ and $\beta$ are defined as moving object distortion coefficients. The YUV values of moving object pixel and background pixel are acquired in YUV color space, then we can calculate the value of $\theta$ which is the distortion coefficient of the moving object for the relative background by the following formula (1).

$$
\theta=\arccos \left(\frac{\mathrm{EB} \cdot \mathrm{EM}}{|\mathrm{EB}| \cdot|\mathrm{EM}|}\right)
$$

The steps of the shadow removal the paper proposed are described as follows:

Step 1. Input the video image frames with YUV format.

Step 2. Calculate the value of $\theta$ which is the distortion coefficient of the moving object for the relative background by formula 1 based on the pixels computing model in YUV space.

Step 3. If $\theta>T_{\theta}\left(T_{\theta}\right.$ means the optimal threshold value), then this pixel point is defined as the actual object; otherwise, this pixel point is defined shadow area.

In this method, the value of $\mathrm{T}_{\theta}$ is relative to $\gamma$ and $\beta$ referred in the above.

It is found in the experiment that if the value of $\theta$ calculated by formula (1) are used directly, the shadow detection results will be very accurate in high pixel brightness and saturation while there is much error between the shadow detection results and the actual results in low pixel brightness and saturation. In YUV color space, the component value of brightness, chromaticity and intensity may not have same effects. On one hand, owning to great difference of brightness between object and shadow, we should decrease appropriately the weight of brightness component and let $\lambda$ denote it; On the other hand, because the chromaticity and intensity component of shadows is equal or nearly the same as background, we should increase the weight of chromaticity and intensity component and let $\eta$ denote it. Taking into account the effect of $\lambda$ and $\eta$, we can calculate the value of $\theta$ which is the distortion coefficient of the moving object for the relative background by the following formula (2) instead of formula (1).

$$
\theta=\arccos \left(\frac{\lambda^{2} \mathrm{EBx} \times \mathrm{EMx}+\eta^{2} \mathrm{EBy} \times \mathrm{EMy}+\eta^{2} \mathrm{EBz} \times \mathrm{EMz}}{\sqrt{\lambda^{2} \mathrm{EBx}^{2}+\eta^{2} \mathrm{EBy}^{2}+\eta^{2} \mathrm{EBz}^{2}} \sqrt{\lambda^{2} \mathrm{EMx}^{2}+\eta^{2} \mathrm{EMy}^{2}+\eta^{2} \mathrm{EMz}^{2}}}\right)
$$

Experimental results show that if we calculate the value of $\theta$ by formula 2, the shadow detection results will also be accurate even in low pixel brightness and saturation. We obtain the optimal parameter values by optimizing experiment as follows: $\lambda=0.33, \eta=0.67, T_{\theta}=4$.

\section{Experimental Results and Analysis}

The video image frame used in the features a size of $640 \times 480$ and $320 \times 240$ in true color format. The operational environment: Intel ${ }^{\circledR}$ Core $^{\mathrm{TM}}$ Duo CPU T6600@2.20GHz,2GB RAM, and Windows XP operating system. We will analyze the experimental results from two aspects as follows.

\subsection{Shadow Detection and Removal}

The processing of shadow detection and removal using usual methods is illustrated as follows ${ }^{[9]}: 1$ ) Background subtraction image is obtained by using the current frame minus the background frame. 2) Binary processing of background subtraction image. 3) Removing shadow. 4) Eliminating the isolated noise point in background area and the loophole in foreground area by morphological filtering and region filling method.5) The moving object are got after the process mentioned above. Fig. 2 is the processing of shadow detection and removal by this paper's method. a): two video frames with moving objects. Bicycle with shadow is No.10 frame in a surveillance video on the left, and pedestrian with shadow is No.15 frame on the right. b): the detection results of object and shadow by the paper's method. The black area denotes background, the white area denotes real object and the gray area denotes object shadow. c): the detection results after removing object shadow. Obviously, the shadows of pedestrian and bicycle are removed effectively. 

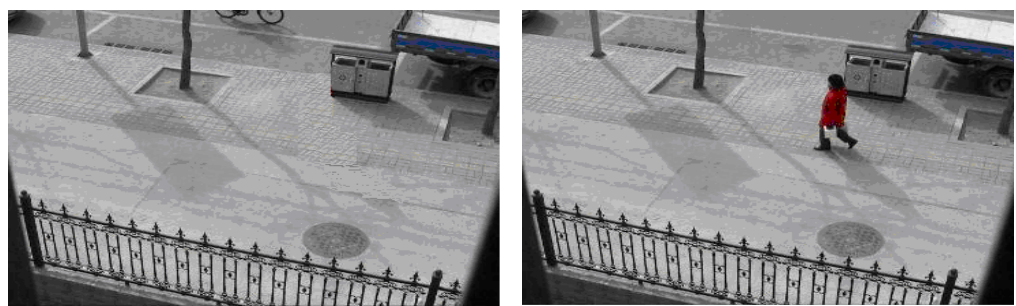

a) two video frames with moving objects
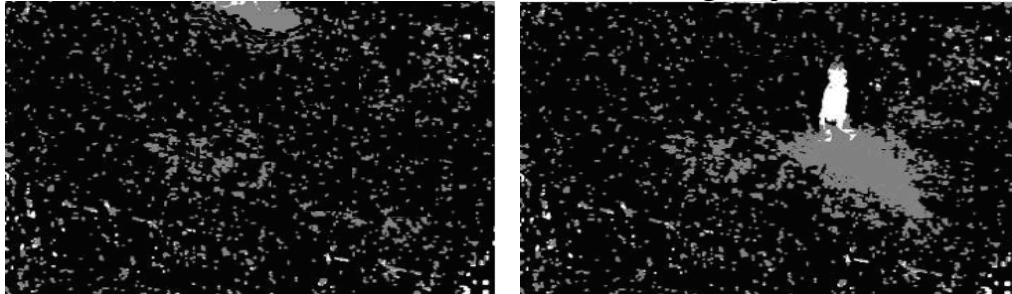

b) the dection results of object and shadow by the paper's method
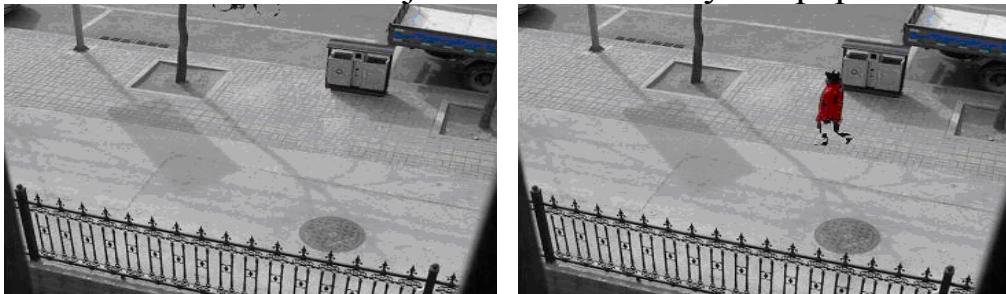

c) the detection results after removing object shadow

Fig. 2 the processing of shadow detection and removal by this paper's method

As shown in Fig.2, we can see that the paper's method can detect moving objects and remove shadows simultaneously in video images.it has the advantage of quick and simple compare with usual methods.

\subsection{Eliminate Object Adhesion}

When shadow of an object is close to the other object, the usual object detection method may mistake the two objects as one. This is called "object adhesion". The paper's method can eliminate object adhesion efficiently. Fig. 3 is the object detection results of No. 523 frame in a surveillance video. a) :the detection results without using any shadow removal method, two cars marked by the blue rectangular box are mistaken as one object; b) :the detection results by the paper's method, it can accurately identify the two cars.

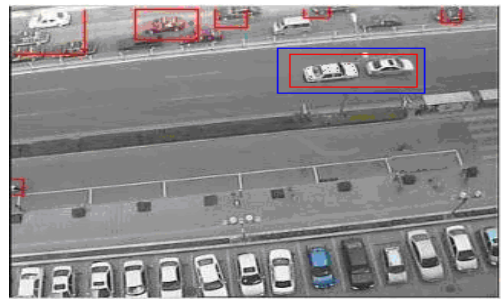

a) the detection results without using any shadow removal method

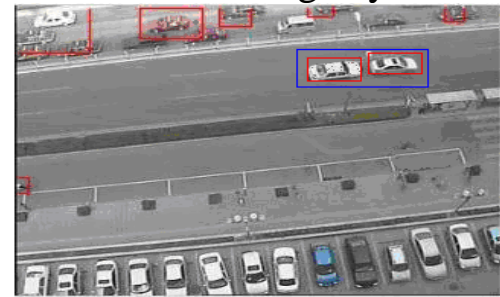

b) the detection results by the paper's method

Figure 3 Comparison of No.523 Frame Detection Results 


\section{Conclusion}

This paper proposes a fast shadow removal method based on video image in YUV color space. The method can detect moving objects and remove shadows simultaneously in video images and simplify the processing of shadow removal by usual method. The experimental results show that the method for shadow removal can quickly remove the shadow and separate several joined objects correctly.

\section{Acknowledgements}

This research was supported in part by the National Spark Program (No.2015GA740054) and the Binzhou University Research Foundation (No. BZXYG1713).

\section{References}

[1]. Zhang Hongying, Li Hong, Sun Yigang. Shadow removal algorithm based on gaussian mixture mode[J]. Journal of Computer Application. Vol. 33(2013) No.1, p.31-34.

[2]. Boroujeni H S, Charkari N M. Robust moving shadow detection with hierarchical mixture of MLP experts [J]. Signal, Image and Video Processing. Vol. 8(2014)No.7, p.1291-1305.

[3]. Adeline K R M, Chen M, Briottet X, et al. Shadow detection in very high spatial resolution aerial images: A comparative study[J]. Isprs Journal of Photogrammetry \& Remote Sensing. Vol. 80(2013) No.142, p.21-38.

[4]. Tiwari S, Chauhan K, Kurmi Y. Shadow Detection and Compensation in Aerial Images using MATLAB[J]. International Journal of Computer Applications. Vol. 119(2015) No.1, p.5-9.

[5]. Gruzman I S. Using gradient tensors of the second and third orders for segmentation of images containing textures with structural redundancy[J]. Optoelectronics, Instrumentation and Data Processing. Vol. 52(2016) No.1, p.17-23.

[6]. Tian B, Morris B T, Tang M, et al. Hierarchical and Networked Vehicle Surveillance in ITS: A Survey[J]. IEEE Transactions on Intelligent Transportation Systems. Vol. 18(2015) No.1, p.25-48.

[7]. Liu Yang, Li Yushan, Zhang Dapu. A chrominance distortion and texture based method for shadow suppression[J].Computer Science ,2005,32(9):211-214.

[8]. Liu D, Yan Y, Shyu M L, et al. Spatio-Temporal Analysis for Human Action Detection and Recognition in Uncontrolled Environments[J]. International Journal of Multimedia Data Engineering \& Management. Vol. 6(2015) No.1, p.1-18.

[9]. Zhang Xia. Method for eliminating moving object shadows in video images[J]. Computer Engineering and Applications. Vol. 49(2013) No.6, p.201-204.

[10]. Wang Jun, Wang Yuehuan, Jiang Man, et al. Moving cast shadow detection using online sub-scene shadow modeling and object inner-edges analysis[J]. Journal of Visual Communication \& Image Representation. Vol. 25(2014) No.5, p.978-993.

[11]. Liu Xue, Chang Faliang, Wang Huajie. An shadow suppression method in detecting moving object[J].Microprocessors,2008,10(5):116-121.

[12]. Xu Weijian, Zhao Caidan, Chiang Huapei, et al. The RR-PEVQ algorithm research based on active area detection for big data applications[J]. Multimedia Tools and Applications. Vol. 74(2015) No.10, p.3507-3520.

[13]. Liu Qing, Qin Xiuli, Wen Songbai,et al. The algorithm of shadow-elimination based on YUV color space and graphy theory[J].CAAI Transactions on Intelligent Systems,2010,5(6):556-560. 